\title{
Suppression of GRASP65 phosphorylation by tetrahydrocurcumin protects against cerebral ischemia/reperfusion injury via ERK signaling
}

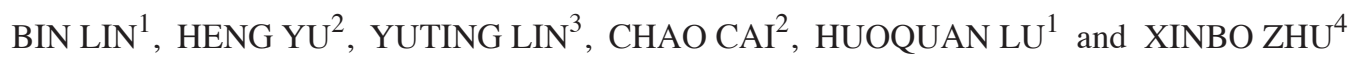 \\ ${ }^{1}$ Department of Pharmacy, Changxing People's Hospital of Zhejiang, Huzhou, Zhejiang 313100; \\ ${ }^{2}$ Department of Medicine, First Clinical Medicine School; ${ }^{3}$ Department of Medicine, Renji School; \\ ${ }^{4}$ Department of Pharmacy, Pharmacy School, Wenzhou Medical University, Wenzhou, Zhejiang 325035, P.R. China
}

Received September 2, 2015; Accepted September 9, 2016

DOI: $10.3892 / \mathrm{mmr} .2016 .5816$

\begin{abstract}
The aim of the present study was to assess the neuroprotective effects of tetrahydrocurcumin (THC) in a mouse model of cerebral ischemia/reperfusion (I/R) injury, and to investigate the involvement of Golgi reassembly and stacking protein 65 (GRASP65) and the extracellular signal-regulated kinase (ERK) signaling pathway. Cerebral I/R injury was induced using the Pulsinelli four-vessel occlusion method. After $5 \mathrm{~min}$ of reperfusion, mice received THC $(5,10$ or $25 \mathrm{mg} / \mathrm{kg})$ or saline by intraperitoneal injection. After $24 \mathrm{~h}$ of reperfusion, mice underwent neurological evaluation. Infarct volumes were determined by triphenyltetrazolium chloride staining, and levels of superoxide dismutase and malondialdehyde were measured in brain tissue homogenates. Expression of GRASP65, phosphorylated-GRASP65, ERK and phosphorylated-ERK was determined by western blotting. THC induced a dose-dependent decrease in the phosphorylation of ERK and GRASP65. Thus, THC attenuated I/R injury-induced activation of the ERK signaling
\end{abstract}

Correspondence to: Professor Huoquan Lu, Department of Pharmacy, Changxing People's Hospital of Zhejiang, 66 Taihu Road, Huzhou, Zhejiang 313100, P.R. China

E-mail: rylhq@126.com

Professor Xinbo Zhu, Department of Pharmacy, Pharmacy School, Wenzhou Medical University, 11 Qiuzhen Building, Chashan Campus, Chashan Street, Wenzhou, Zhejiang 325035, P.R. China E-mail: xinbozhu@163.com

Abbreviations: THC, tetrahydrocurcumin; ERK, extracellular signal-regulated kinase; GRASP, golgi reassembly and stacking proteins; Cur, curcumin; SOD, superoxide dismutase; MDA, malondialdehyde; ROS, reactive oxygen species; TTC, tetrzolium chloride

Key words: Golgi apparatus stress, cerebral ischemia reperfusion injury, tetrahydrocurcumin, extracellular signal-regulated kinase, golgi reassembly and stacking proteins pathway and reduced the phosphorylation of GRASP65. THC exhibited a dose-dependent protective effect against cerebral I/R injury, mediated by suppression of the ERK signaling pathway and a subsequent reduction in GRASP65 phosphorylation. The current study provided new information in the research of the cerebral ischemia-reperfusion injury mechanism.

\section{Introduction}

Cerebral ischemia/reperfusion (I/R) injury occurs as a complication of stroke following thrombolysis and a recurrence of ductal patency. The mechanisms of I/R injury are not clearly understood, and identification of effective and safe treatments for ischemic stroke is required.

The Golgi apparatus is an organelle essential for protein synthesis and maturation. In response to conditions of stress, including physiological imbalances or disruption of cell morphology, the transcription of Golgi-associated genes can be upregulated to restore homeostasis or induce apoptosis; this mechanism is termed the Golgi stress response $(1,2)$. Research suggests the extracellular signal-regulated kinase (ERK) signaling pathway is involved in the response to oxidative stress. A previous study demonstrated that the ERK signaling pathway regulates phosphorylation of the Golgi reassembly and stacking protein 65 (GRASP65), resulting in Golgi cisternal unstacking (3).

Curcumin (Cur), a yellow dye in the crude drug 'Turmeric' (Curcumae rhizoma) from the rhizome of Curcuma longa L., is reported to have anti-inflammatory, anti-oxidative and anti-tumor effects (4-6). Tetrahydrocurcumin (THC) is an active metabolite of Cur, and has been identified in human and rat intestinal mucosa, and in hepatic cytosol (7). THC and Cur have identical $\beta$-diketone structures and phenolic groups, but differ in that THC lacks the double bonds of Cur (Fig. 1). Recent research suggests that THC exerts greater anti-oxidant activity than Cur in certain in vitro and in vivo systems (8-10).

The present study examined the protective effects of THC against cerebral I/R injury in mice, and reviewed the mechanisms of Golgi stress-induced cerebral I/R injury via the ERK signaling pathway. 


\section{Materials and methods}

Animals. Male specific pathogen-free ICR mice ( $\mathrm{n}=100$; 2 months old; 23-27 g) were provided by the Experimental Animal Center of Wenzhou Medical University (Wenzhou, China). Mice had ad libitum access to food and water and were housed in temperature- and humidity-controlled conditions (temperature; $22 \pm 1^{\circ} \mathrm{C}$; humidity $56 \pm 5 \%$ ) with a 12 -h light/dark cycle. All mice were treated humanely, and the study was approved by the Experimental Animal Ethics Committee of Wenzhou Medical University.

Chemicals. THC was purchased from Dalian Meilun Biotech Co., Ltd. (Dalian, China). Sodium chloride injections (0.9\%) were purchased from Hangzhou Minsheng Pharmaceutical Group Co., Ltd. (Hangzhou, China). Dimethyl sulfoxide (DMSO), chloral hydrate (10\%; Sinopharm Chemical Reagent Co., Ltd., Shanghai, China) and 2,3,5-triphenyl-2H-tetrazolium chloride (TTC) were purchased from Sigma-Aldrich (Merck Millipore, Darmstadt, Germany). Paraformaldehyde (4\%) was provided by the Experimental Neural Organisms Institution of Wenzhou Medical University (Wenzhou, China). Total superoxide dismutase (T-SOD) kits, lipid peroxidation [malondialdehyde (MDA)] assay kits, total protein kits and bicinchoninic acid (BCA) protein assay kits were purchased from Nanjing Jiancheng Bioengineering Institute (Nanjing, China). Anti-GR ASP65 (sc-398363) and anti-phospho (p)-GRASP65 (sc-389542) antibodies were purchased from Santa Cruz Biotechnology, Inc. (Dallas, TX, USA), and anti-ERK (4696S) and anti-pERK (9106L) antibodies were purchased from Cell Signaling Technology, Inc. (Danvers, MA, USA).

Animal surgery and THC administration. The mice were randomly divided into 5 groups $(\mathrm{n}=20$ per group) as follows: Sham-operated controls (group A), I/R group (group B), low-dose THC (5 mg/kg; group C1), moderate-dose THC (10 mg/kg; group C2) and high-dose THC (25 mg/kg; group $\mathrm{C} 3)$.

Cerebral ischemia was induced by Pulsinelli's four-vessel occlusion method (11). All surgeries were performed by the same person. Under anesthesia with $10 \%$ chloral hydrate ( $3 \mathrm{mg} / \mathrm{kg}$ ), a $1.5 \mathrm{~cm}$ incision was made in the middle of the neck and the jugular muscles were separated. The bilateral common vertebral arteries were identified and occluded by electrocoagulation. The incision was sutured and the animals were allowed to recover. The following day, the procedure was repeated and the common carotid arteries were occluded for 5 min using vitreous needles and small bulldog clamps. Sham-operated animals underwent the same surgical procedure without occlusion. Treatment injections were administered after $5 \mathrm{~min}$ of reperfusion. Groups A and B received intraperitoneal (i.p.) injections of equal volumes of normal saline. A $2 \%$ THC solution was prepared in DMSO; groups $\mathrm{C} 1, \mathrm{C} 2$ and $\mathrm{C} 3$ received i.p. injections of THC at a low $(5 \mathrm{mg} / \mathrm{kg})$, moderate $(10 \mathrm{mg} / \mathrm{kg})$, or high $(25 \mathrm{mg} / \mathrm{kg})$ dose, respectively.

Neuroethological assessment. After $24 \mathrm{~h}$ of reperfusion, mice were evaluated for exponents of stroke (Table I) and neurological symptoms (Table II) (12).

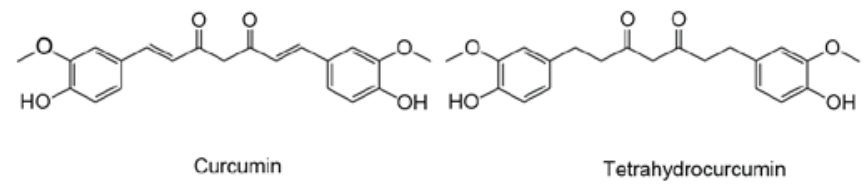

Figure 1. Chemical structures of curcumin and tetrahydrocurcumin.

Specimen collection and preparation of pathological sections. After $24 \mathrm{~h}$ of reperfusion, mice were anesthetized with $10 \%$ chloral hydrate prior to decapitation, then were transcardially perfused with normal saline. The brain was dissected via craniotomy and fixed in a $4 \%$ paraformaldehyde solution for $24 \mathrm{~h}$ prior to conventional paraffin embedding. The specimens were sectioned coronally (behind the optic chiasm) at a thickness of $5 \mu \mathrm{m}$. Sections were stained with hematoxylin and eosin (HE), as previously described (13).

TTC staining. Mice were decapitated after $24 \mathrm{~h}$ of reperfusion. The brain was removed and cooled in normal saline at $4^{\circ} \mathrm{C}$ for $10 \mathrm{~min}$. The specimens were sectioned coronally into four 2-mm-thick slices using a brain matrix. Slices were incubated with a $2 \%$ aqueous solution of TTC in the dark for $30 \mathrm{~min}$ at $37^{\circ} \mathrm{C}$ in a water bath, and then images using a digital camera (Canon 600D; Canon, Inc., Tokyo, Japan). Unstained areas were defined as infarcted and measured using image analysis software (Image-Pro Plus, version 6.0; Media Cybernetics, Inc., Rockville, MD, USA). Total infarct volume in the brain was calculated as the sum of the infarct volumes of the four brain slices (14).

Assessment of cerebral SOD and MDA. Cerebral SOD and MDA levels were measured in brain tissue homogenates prepared with a $0.9 \%$ saline solution and centrifuged at $1,006 \mathrm{x} g$ for $10 \mathrm{~min}$ at $4^{\circ} \mathrm{C}$. Supernatants were collected and analyzed using the SOD and MDA kits. Concentrations of SOD and MDA were calculated based on the optical density readings obtained at 550 and $552 \mathrm{~nm}$, respectively, using the Multiskan Spectrum microplate reader (Thermo Fisher Scientific, Inc., Waltham, MA, USA).

Western blot analysis of ERK, pERK, GRASP65 and $p$ GRASP65. ERK, pERK, GRASP65 and pGRASP65 levels in brain tissue homogenates were determined by western blotting. Protein extraction kits from were purchased from Jiancheng Bioengineering Institute (Nanjing, China). Total protein levels in the homogenate samples were determined using the BCA protein assay kit. Equal amounts of protein (20 $\mu \mathrm{g}$ sample/lane) from each sample were then subjected to $10 \%$ SDS-PAGE. The gel was transferred to a a polyvinylidene difluoride membrane, and proteins were detected with antibodies against ERK (1:1,000), pERK (1:1,000), GRASP65 (1:200), and pGRASP65 (1:500). $\beta$-actin (1:4,000; Santa Cruz Biotechnology, Inc.) was used as a loading control. Subsequently, the membranes were incubated with horseradish peroxidase-conjugated anti-rabbit (KS001) or anti-goat (KS003) secondary antibodies (dilution, 1:3,000) for $2 \mathrm{~h}$ at $24^{\circ} \mathrm{C}$, and were visualized using Western Blotting Chemiluminescence Reagent, followed by exposure to X-ray films. Blots were quantified using BandScan software (version 5.0; Glyko, Inc., Novato, CA, USA). 
Table I. Exponents of stroke.

\begin{tabular}{lc}
\hline Symptom & Score \\
\hline Messy fur, tremor & 1 \\
Decreasing exercise or bovine movement & 1 \\
Bovine feeling of ears & 3 \\
Turned up head & 3 \\
Eyes opened & 3 \\
Posterior limb shaped like Chinese character eight (八) & 3 \\
Upper eyelid drooping & 1 \\
Circling & 3 \\
Eclampsia or paroxysmal movement & 3 \\
Exceedingly weak & 6 \\
\hline
\end{tabular}

The total score is 25 and divided into three categories: Symptom (score, $0-3$ ), possible injury (score, $3-9$ ), obvious injury (score, $\geq 10$ ).

Table II. Neurological symptom assessment.

\begin{tabular}{lc}
\hline Symptom & Score \\
\hline Idiopathic excavation & 0 \\
Walking while being stimulated & 1 \\
Cannot move & 2 \\
Normal gait & 0 \\
Ataxia & 1 \\
Crawling & 2 \\
No gait & 3 \\
Able to feed & 0 \\
Cannot feed & 1 \\
Able to drink & 0 \\
Cannot drink & 1 \\
Removable to pains & 0 \\
Only head or trunk can move & 1 \\
Limbs can retrace or no reaction & 3 \\
\hline
\end{tabular}

The total score is 15 , and divided into four categories: Normal (score, 0 ), mild injury (score, 1-3), moderate injury (score, 4-6), severe injury (score, 7-15).

Statistical analysis. Statistical comparisons were made using analysis of variance (ANOVA) followed by post-hoc Bonferroni tests. Data are presented as the mean \pm standard devation. Neuroethological and stroke assessment data were analyzed using the Friedman test. Friedman two-way ANOVA was used for multiple comparisons. Statistical analyses were performed using SPSS software (version 19.0; IBM SPSS, Armonk, NY, USA). $\mathrm{P}<0.05$ were considered to indicate a statistically significant difference.

\section{Results}

Neuroethological assessment following cerebral I/R. After $24 \mathrm{~h}$ of reperfusion, various neurological symptoms were

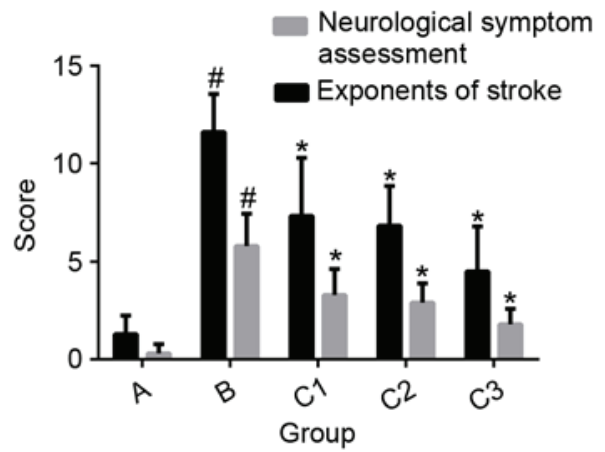

Figure 2. Neuroethological assessment of the mice in each group following I/R for $24 \mathrm{~h}$. ${ }^{\#} \mathrm{P}<0.01$ vs. group $\mathrm{A},{ }^{*} \mathrm{P}<0.01$ vs. group $\mathrm{B}$. I/R, ischemia/reperfusion; Group A, sham; Group B, I/R control; Group C1, low-dose tetrahydrocurcumin; Group C2, moderate-dose tetrahydrocurcumin; Group C3, high-dose tetrahydrocurcumin.

observed: Decreased exercise, lack of feeding, bovine-like reaction, ataxia (in some mice), circling with the tail lifted, posterior limbs positioned as the Chinese character for eight (八), upper eyelid drooping or inability to open the eyes, and messy fur. Compared with group B (normal saline-injected mice), the exponents of stroke and neurological assessment scored were significantly decreased in the THC-treated groups ( $\mathrm{P}<0.01$; Fig. 2).

Effect of THC on ischemic neuronalnecrosis. In sham-operated mice (group A), cells were closely arranged, regularly shaped and uniformly stained by HE (Fig. 3). By contrast, cells from mice with I/R injury (group B) were loosely arranged with various abnormal pathological changes, including karyopyknosis, interstitial edema, neuronal degeneration and vacuolization. Cells from mice administered the high dose of THC (25 mg/kg; group C3) were mostly normal, though karyopyknosis was evident in some cells (Fig. 3). Cells from mice administered the low dose of THC ( $5 \mathrm{mg} / \mathrm{kg}$; group $\mathrm{C} 1)$ exhibited more severe apoptosis, with higher levels of karyopyknosis, irregular arrangement, interstitial edema, neuronal degeneration and vacuolization compared with group C3. In mice administered the moderate dose of THC $(10 \mathrm{mg} / \mathrm{kg}$; group C2), the cellular effects were between those observed in groups $\mathrm{C} 1$ and $\mathrm{C} 3$ (Fig. 3).

Effect of THC on infarct size following I/R injury. After $24 \mathrm{~h}$ of reperfusion, extensive infarction was evident in group B mice. The infarct size was significantly reduced in all THC treatment groups compared with group B ( $\mathrm{P}<0.01$; Figs. 4 and 5), and the effect was dose-dependent. No infarction was observed in the sham-operated group.

Effect of THC on SOD and MDA. Compared with group B, mice administered THC exhibited significantly increased levels of SOD $(\mathrm{P}<0.01)$ and lower levels of MDA in brain tissue homogenates $(\mathrm{P}<0.01$; Fig. 6). The effects of THC on SOD and MDA were dose-dependent.

Effect of THC on the expression of ERK, pERK, GRASP65 and $p$ GRASP65. Western blot analysis demonstrated the protein expression of ERK in ischemic brain tissue did not 


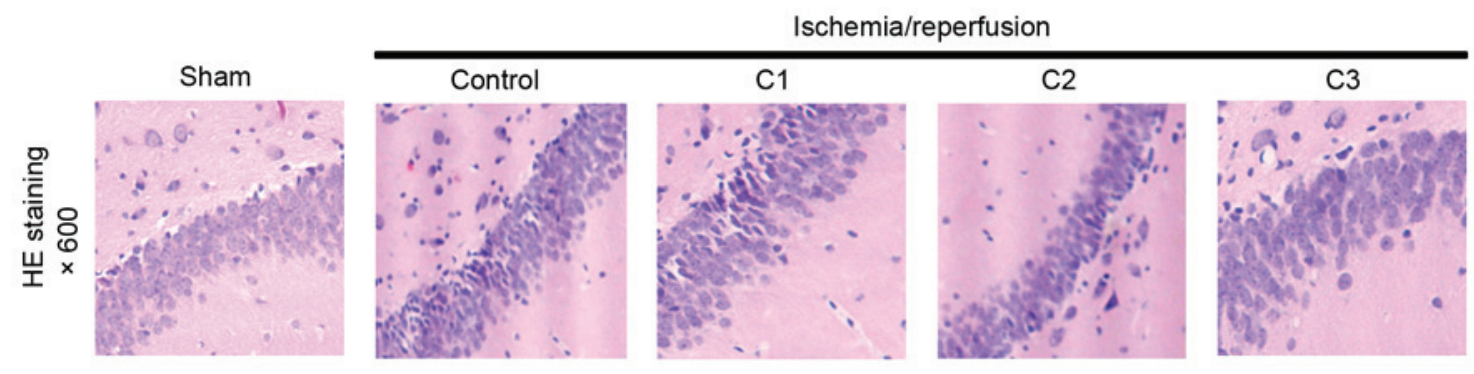

Figure 3. Changes in the neurocyte morphology of cerebral ischemia/reperfusion injury mice. Stained with HE. Magnification, x600. HE, hematoxylin and eosin; $\mathrm{C} 1$, low-dose tetrahydrocurcumin; $\mathrm{C} 2$, moderate-dose tetrahydrocurcumin; C3, high-dose tetrahydrocurcumin.

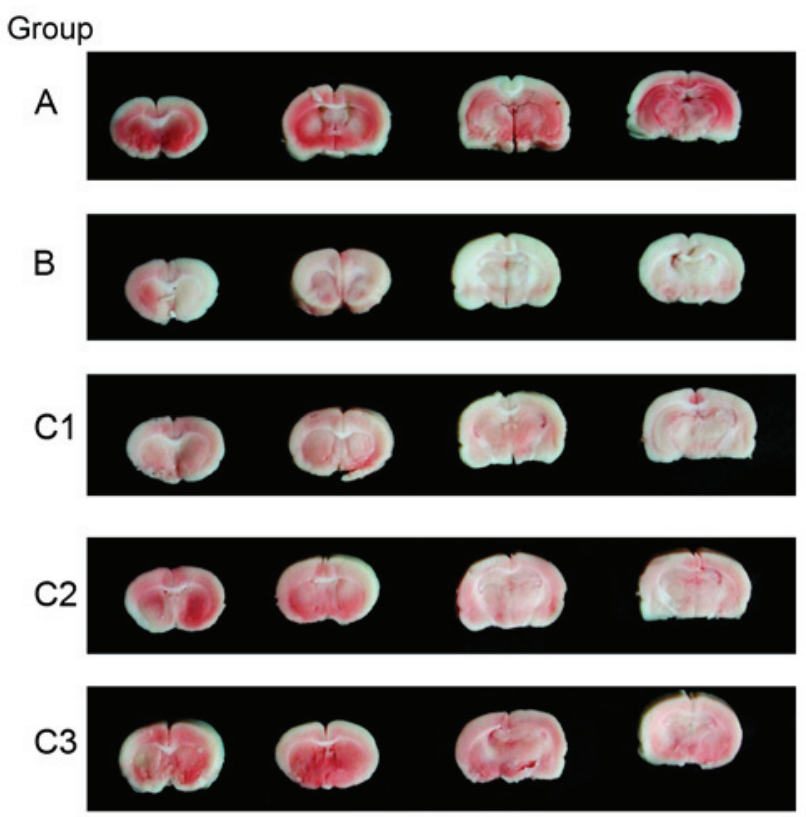

Figure 4. Infarct size of brain tissue following reperfusion for $24 \mathrm{~h}$ stained with triphenyltetrazolium chloride. Group A, sham; Group B, control; Group C1, low-dose tetrahydrocurcumin; Group C2, moderate-dose tetrahydrocurcumin; Group C3, high-dose tetrahydrocurcumin.

differ the between groups ( $\mathrm{P}>0.05$; Fig. 7). However, the level of pERK and the pERK/ERK ratio were significantly increased in group B compared with group A $(\mathrm{P}<0.01 ;$ Fig. 7$)$. The $\mathrm{pERK} / \mathrm{ERK}$ ratio as significantly reduced in groups $\mathrm{C} 1$ $(\mathrm{P}<0.05), \mathrm{C} 2(\mathrm{P}<0.01)$ and $\mathrm{C} 3(\mathrm{P}<0.01)$ compared with group $\mathrm{B}$ (Fig. 7). This suggests that the ERK signaling pathway was suppressed by THC.

Expression of total GRASP65 did not differ significantly between groups ( $P>0.05$; Fig. 7). However, the level of pGRASP65 was significantly increased in group $\mathrm{B}$ compared with group $\mathrm{A}(\mathrm{P}<0.01$; Fig. 7). In groups $\mathrm{C} 1, \mathrm{C} 2$ and $\mathrm{C} 3$, pGRASP65 was significantly reduced compared with group B (P<0.01; Fig. 7).

The results of the present study suggest that the upregulated phosphorylation of GRASP65 following I/R injury may be due to, at least partially, the increased activation of the ERK signaling pathway induced by THC. Thus, THC protects against cerebral I/R injury via suppression of GRASP65 phosphorylation, and this effect may be mediated by the ERK signaling pathway.

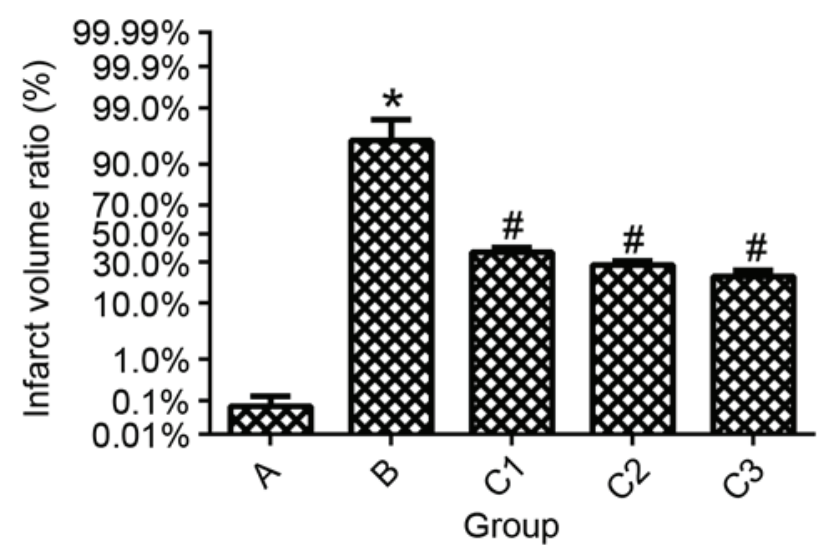

Figure 5. Infarct volume ratio of the brain tissue in each group following reperfusion for $24 \mathrm{~h}$. ${ }^{*} \mathrm{P}<0.01$ vs. Group A, ${ }^{~} \mathrm{P}<0.01$ vs. Group B. Data are presented as the mean \pm standard deviation; $n=10$. Group A, sham; Group B, control; Group C1, low-dose tetrahydrocurcumin; Group C2, moderate-dose tetrahydrocurcumin; Group C3, high-dose tetrahydrocurcumin.

\section{Discussion}

The ERK signaling pathway has an important role in cerebral I/R injury. ERK is involved in growth and differentiation, mediated by growth factor receptors, and is also activated by oxidative stress, cerebral ischemia and the release of neurotransmitters under certain pathological conditions $(15,16)$. In the current study, the level of pERK and the pERK/ERK ratio were significantly increased in ischemic mice compared with sham-operated controls, indicating that the ERK pathway was activated in response to cerebral I/R injury. In mice administered THC, pERK was significantly reduced compared with the control, suggesting that THC inhibited I/R injury-induced activation of the ERK pathway. This effect was dose-dependent, which provides further evidence of the neuroprotective properties of THC.

GRASPs are membrane proteins involved in Golgi stacking. They regulate Golgi assembly, and cell migration, division and apoptosis. Specifically, GRASP65 mediates Golgi morphological changes during pathophysiological conditions, and is involved in cell division and apoptosis (17-21).

In the present study, expression of GRASP65 and pGRASP65 were elevated following cerebral I/R injury compared with sham-operated animals $(\mathrm{P}<0.01)$. Previous research has demonstrated that GRASP65 is phosphorylated 

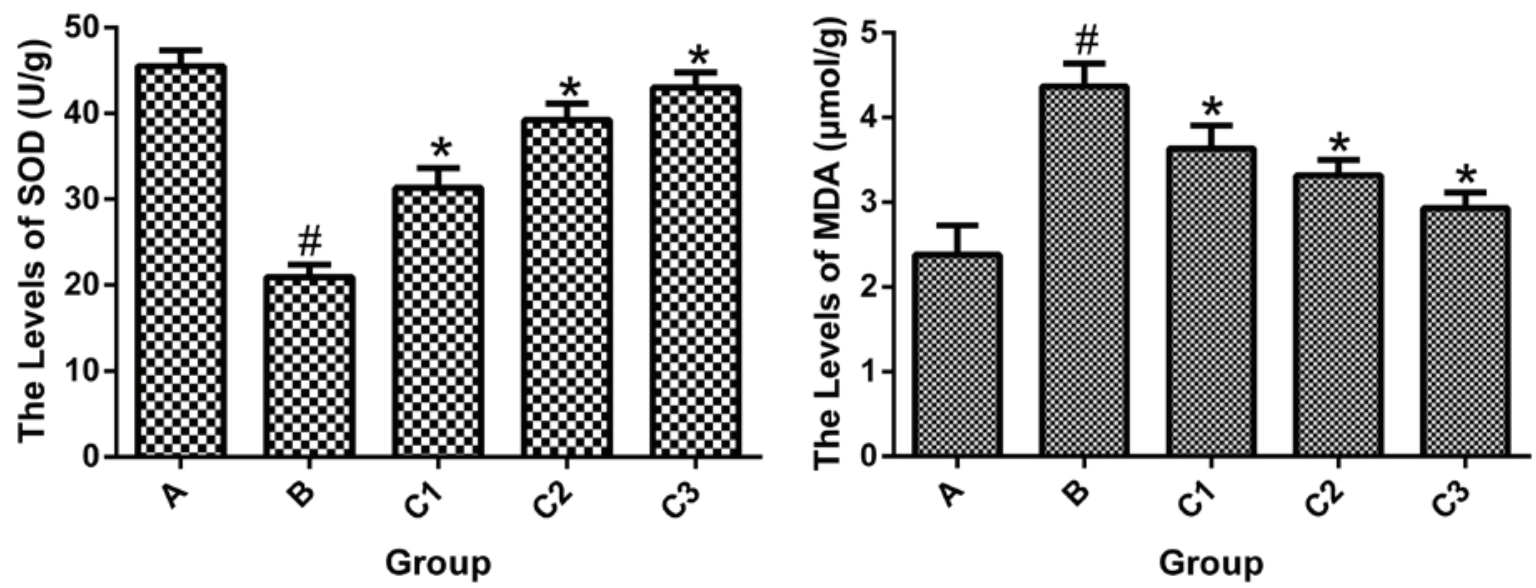

Figure 6. Changes in the levels of SOD and MDA in mice cerebral tissue homogenates. ${ }^{~} \mathrm{P}<0.01$ vs. Group $\mathrm{A}$, $\mathrm{P}<0.01$ vs. Group B. Data are presented as the mean \pm standard deviation; $\mathrm{n}=10$. SOD, superoxide dismutase; MDA, malondialdehyde; Group A, sham; Group B, control; Group C1, low-dose tetrahydrocurcumin; Group C2, moderate-dose tetrahydrocurcumin; Group C3, high-dose tetrahydrocurcumin.
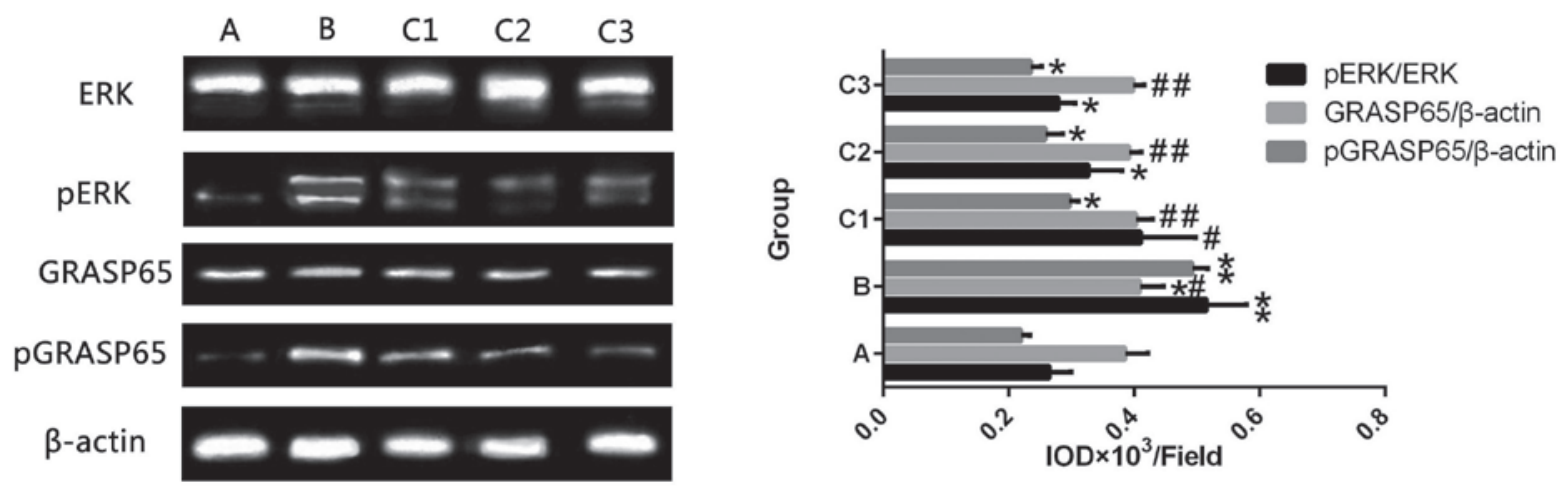

Figure 7. ERK, pERK, GRASP 65 and pGRASP 65 expression in mice from each group following reperfusion for $24 \mathrm{~h} .{ }^{* *} \mathrm{P}<0.01$ vs. Group A, ${ }^{*} \mathrm{P}>0.05$ vs. Group A, "P<0.05 vs. Group B, "P<0.01 vs. Group B, ${ }^{\# \# ~} \mathrm{P}>0.05$ vs. Group B. Data are presented as the mean \pm standard deviation; $\mathrm{n}=10$. Group A, sham; Group B, control; Group C1, low-dose tetrahydrocurcumin; Group C2, moderate-dose tetrahydrocurcumin; Group C3, high-dose tetrahydrocurcumin; ERK, extracellular-signal regulated kinase; p, phosphorylated; GRASP65, Golgi reassembly and stacking protein 65.

by ERK, cyclin dependent kinase 1 and polo like kinase 1 during cell division, which leads to depolymerization and division of the Golgi (17,18,20-22).

The ERK signaling pathway may be involved in neuronal degeneration following I/R injury. In mice treated with THC, a dose-dependent decrease in the phosphorylation of GRASP65 was observed, and this effect may be mediated by ERK. The Golgi is essential for the endoplasmic reticulum and mitochondria during conditions of oxidative stress as a downstream target organelle associated with phosphorylation of GRASP65. The Golgi stress response suppresses the synthesis of required proteins, and thereby impacts the extent of $\mathrm{I} / \mathrm{R}$ injury.

Reactive oxygen species (ROS) are produced as a result of incomplete reduction of oxygen within the electron transport chain. Examples of ROS include superoxide anion, singlet oxygen, hydrogen peroxide and hydroxyl radicals. These ROS typically function as signaling molecules, however during stress, they can lead to cell damage and tissue necrosis. Anti-oxidant enzymes, including glutathione reductase, catalase and SOD, are effective at neutralizing ROS. During $\mathrm{I} / \mathrm{R}$ injury, SOD is involved in maintaining homeostasis and minimizing the damaging effects of ROS (23-25). MDA is an end product of lipid peroxidation in cells, and increased levels of ROS can trigger overproduction of MDA. SOD and MDA are often used as markers of oxidative stress and anti-oxidant status, respectively. Cur is reported to have anti-oxidative properties and a neuroprotective effect against cerebral I/R injury (26). For example, Wang et al demonstrated that Cur attenuates oxidative stress injury induced by hypoxic-ischemic brain damage in rats. In the present study, mice treated with THC exhibited increased levels of SOD following I/R injury compared with mice given saline, and this effect was dose-dependent. Levels of MDA were increased following I/R injury, and THC attenuated this effect in a dose-dependent manner. The evaluation of neurological behavior revealed a significant dose-dependent effect of THC.

In summary, THC had a dose-dependent protective effect against cerebral I/R injury, mediated by suppression of the ERK signaling pathway and a subsequent reduction of GRASP65 phosphorylation. The current study investigated the cerebral ischemia-reperfusion injury mechanism, and suggested that THC may be a potential therapeutic agent for the prevention of ischemic brain injury. 


\section{Acknowledgements}

This project was supported by funding from the National Students' Innovation and Entrepreneurship Training Program (grant no. 201410343003).

\section{References}

1. Li T, You H, Zhang J, Mo X, He W, Chen Y, Tang X, Jiang Z, Tu R, Zeng L, et al: Study of GOLPH3: A potential stress-inducible protein from Golgi apparatus. Mol Neurobiol 49: 1449-1459, 2014.

2. Li T, You H, Mo X, He W, Tang X, Jiang Z, Chen S, Chen Y, Zhang J and Hu Z: GOLPH3 mediated golgi stress response in modulating N2A cell death upon oxygen-glucose deprivation and reoxygenation injury. Mol Neurobiol 53: 1377-1385, 2016.

3. Yao Z and Seger R: The ERK signaling cascade - views from different subcellular compartments. Biofactors 35: 407-416, 2009.

4. Chong L, Zhang W, Nie Y, Yu G, Liu L, Lin L, Wen S, Zhu L and $\mathrm{Li} \mathrm{C}$ : Protective effect of curcumin on acute airway inflammation of allergic asthma in mice through Notch1-GATA3 signaling pathway. Inflammation 37: 1476-1485, 2014.

5. García-Niño WR, Zatarain-Barrón ZL, Hernández-Pando R, Vega-García CC, Tapia E and Pedraza-Chaverri J: Oxidative stress markers and histological analysis in diverse organs from rats treated with a hepatotoxic dose of Cr (VI): Effect of curcumin. Biol Trace Elem Res 167: 130-145, 2015.

6. Ji JL, Huang XF and Zhu HL: Curcumin and its formulations: Potential anti-cancer agents. Anticancer Agents Med Chem 12: 210-218, 2012.

7. Naito M, Wu X, Nomura H, Kodama M, Kato Y, Kato Y and Osawa T: The protective effects of tetrahydrocurcumin on oxidative stress in cholesterol-fed rabbits. J Atheroscler Thromb 9: 243-250, 2002.

8. Song KI, Park JY, Lee S, Lee D, Jang HJ, Kim SN, Ko H, Kim HY, Lee JW, Hwang GS, et al: Protective effect of tetrahydrocurcumin against cisplatin-induced renal damage: In vitro and in vivo studies Planta Med 81: 286-291, 2015.

9. Muthumani M and Miltonprabu S: Ameliorative efficacy of tetrahydrocurcumin against arsenic induced oxidative damage dyslipidemia and hepatic mitochondrial toxicity in rats. Chem Biol Interact 235: 95-105, 2015.

10. Sangartit W, Kukongviriyapan U, Donpunha W, Pakdeechote P, Kukongviriyapan V, Surawattanawan P and Greenwald SE: Tetrahydrocurcumin protects against cadmium-induced hypertension, raised arterial stiffness and vascular remodeling in mice. PloS One 9: e114908, 2014.

11. Pulsinelli WA and Brierley JB: A new model of bilateral hemispheric ischemia in the unanesthetized rat. Stroke 10: 267-272, 1979

12. Wei W, Wu XM and Li YJ: Experimental Methodology of Pharmacology. 4th edition. People's Medical Publishing House, Beijing, pp1000-1003, 2010.
13. Chang YH, Fan XN and Shi XM: HE staining and light microscope observation method used in researches of brain tissue. Liaoning J Trad Chin Med 5: 777-779, 2010.

14. Bederson JB, Pitts LH, Germano SM, Nishimura MC, Davis RL and Bartkowski HM: Evaluation of 2,3,5-triphenyltetrazolium chloride as a stain for detection and quantification of experimental cerebral infarction in rats. Stroke 17: 1304-1308, 1986.

15. Zhan L, Li D, Liang D, Wu B, Zhu P, Wang Y, Sun W and $\mathrm{Xu}$ E: Activation of Akt/FoxO and inactivation of MEK/ERK pathways contribute to induction of neuroprotection against transient global cerebral ischemia by delayed hypoxic postconditioning in adult rats. Neuropharmacology 63: 873-882, 2012.

16. Wang S, Wei H, Cai M, Lu Y, Hou W, Yang Q, Dong H and Xiong L: Genistein attenuates brain damage induced by transient cerebral ischemia through up-regulation of ERK activity in ovariectomized mice. Int J Biol Sci 10: 457-465, 2014.

17. Veenendaal T, Jarvela T, Grieve AG, van Es JH, Linstedt AD and Rabouille C: GRASP65 controls the cis Golgi integrity in vivo. Biol Open 3: 431-443, 2014.

18. Ji G, Ji H, Mo X, Li T, Yu Y and Hu Z: The role of GRASPs in morphological alterations of Golgi apparatus: Mechanisms and effects. Rev Neurosci 24: 485-497, 2013.

19. Lane JD, Lucocq J, Pryde J, Barr FA, Woodman PG, Allan VJ and Lowe M: Caspase-mediated cleavage of the stacking protein GRASP65 is required for Golgi fragmentation during apoptosis. J Cell Biol 156: 495-509, 2002.

20. Wang Y, Seemann J, Pypaert M, Shorter J and Warren G: A direct role for GRASP65 as a mitotically regulated Golgi stacking factor. EMBO J 22: 3279-3290, 2003.

21. Wang Y, Satoh A and Warren G: Mapping the functional domains of the Golgi stacking factor GRASP65. J Biol Chem 280: 4921-4928, 2005.

22. Yoshimura S, Yoshioka K, Barr FA, Lowe M, Nakayama K, Ohkuma S and Nakamura N: Convergence of cell cycle regulation and growth factor signals on GRASP65. J Biol Chem 280: 23048-23056, 2005.

23. Niizuma K, Yoshioka H, Chen H, Kim GS, Jung JE, Katsu M, Okami N and Chan PH: Mitochondrial and apoptotic neuronal death signaling pathways in cerebral ischemia. Biochim Biophys Acta 1802: 92-99, 2010.

24. Liu P, Zhao H, Wang R, Wang P, Tao Z, Gao L, Yan F, Liu X, Yu S, Ji X and Luo Y: MicroRNA-424 protects against focal cerebral ischemia and reperfusion injury in mice by suppressing oxidative stress. Stroke 46: 513-519, 2015.

25. Xin X, Li L, Miao L, Zhang QY and Zhu YZ: P105 S-propargyl-cysteine (ZYZ-802), a novel water-soluble donor of endogenous hydrogen sulfide, attenuates impairment against cerebral ischemia-reperfusion injury in rats. Nitric Oxide 39 (Suppl): S47-S48, 2014.

26. Wang JX and Zhu XB: Effects of curcumin on contents of SOD and MDA in brain of neonatal rats with hypoxic-ischemic encephalopathy. Zhong Guo Yao Li Xue Tong Bao Bian Ji Bu 9: 1327-1328, 2013. 